stationary and ergodic processes, illustrating them by means of suitable examples. In fact, one of the great virtues of the book from the point of view of the student approaching the subject in complete ignorance is the large number and relevance of the illustrative examples.

These principles are applied to the derivation of a number of fundamental circuit aspects of noise; derivations which, perhaps in an over-zealous attempt to be straightforward, become rather laboured. The application to practical electrical devices gives a good insight into the noise behaviour of a number of physical devices, in the process of illustrating the application of the theory. This section is naturally far from exhaustive, and this point might have been better brought out if a wider variety of physical systems had been considered; the only devices mentioned are thermionic valves; no mention is made of noise in transistors or other solid-state devices. The section dealing with measurement of noise is, unfortunately, far too brief to do justice to the topic, but as this is essentially a theoretical book this is not of major importance.

The book develops with further theorems related to noise figures and the calculation of optimum arrangements for minimizing them. The reason for this is fully explained in the following chapter which, in great detail, enunciates the problem of detecting ever smaller signals in the presence of noise, stressing the close connexion between accuracy and time taken for an observation.

The remaining section of the book deals with the theoretical aspects of the characteristic function, leading to an analysis of the detection of alternating signals in the presence of noise by means of a non-linear detecting element.

The reason for the final chapter in the book, dealing with target noise, is seen in the author's personal interest in this field. As a consequence, its irrelevant inclusion in a book dealing primarily with the fundamental theory of noise may be excused.

The book thus well comes up to the author's aim of providing a simple introduction to the techniques of noise calculation. From this point of view it is probably the best book yet available for the student and yet one which will be valuable for reference at a later date.

R. A. KrNG

\section{CONVEX GEOMETRY}

\section{Convexity}

By Dr. H. G. Eggleston. (Cambridge Tracts in Mathematics and Mathematical Physics.) Pp. viii + 136. (Cambridge: At the University Press, 1958.) 21s. net.

Problems in Euclidean Space

Application of Convexity. By Dr. H. G. Eggleston. (The Adams Prize Essay of the University of Cam. bridge, 1955-6.) (Intermational Series of Monographs on Pure and Applied Mathematics, Vol. 5.) Pp. viii +165 . (London and New York: Pergamon Press, 1957.) 40s, net.

7 HE basic concept of convexity is simple but powerful. For example, given an ordinary function the graph of which is convex, so that any arc is below its chord, if arbitrary positive masses are attached to a finite number of points, the centroid of such masses is obviously above the curve; but this almost trivial observation can be made to yield most of the significant elementary inequalities of analysis, such as the Cauchy-Hölder inequality. Again, a set of points is convex if, when $A$ and $B$ belong to the set, so do all the points of the straight line segment $A B$. Since many elementary geometrical figures are convex, the question of the relation of convexity to properties of such figures at once arises, leading, for example, to the curious and important notion of curves of constant width.

Dr. H. G. Eggleston's two books are complementary. In the Cambridge Tract, the object is to develop the general theory of convexity, for its own sake, in $n$-dimensional Euclidean space; in the Pergamon Press volume, which consists of the Adams Prize Essay in the University of Cambridge for 1955-56, convexity is the thread connecting a variety of geometrical problems in Euclidean space of two or three dimensions.

A natural description of a plane convex set would be that it lies wholly on one side of any tangent to its boundary, but this is too restrictive (it would rule out a triangle); more generally and more precisely, through every point on the boundary of a convex set there passes a line which does not intersect the set. This provides a convenient and powerful alternative definition of convexity, once its equivalence to the earlier definition is established. Two other valuable tools are the relation between convex sets and convex functions, and Blaschke's selection theorem, which asserts that an infinite aggregate of closed convex sets in a bounded space must contain a sequence converging to a limit set which is closed, convex and not vacuous. All this basic theory is developed for $n$-space in the first four chapters of the Cambridge Tract. The fifth chapter concerns Minkowski's definition of surface area (the volume is simply the appropriate Lebesgue measure); this is a somewhat difficult chapter, for the motivation will scarcely be clear to the general reader. The final two chapters deal with typical geometrical problems : isoperimetrical properties and curves of constant width.

The second book is divided into four sections. In the first, convexity is merely a tool for the investigation of results in point-set theory ; in the second, Borsuk's conjecture that any set of diameter $d$ in $n$-space is the union (sum) of $n+1$ sets each of diameter less than $d$ is proved for 3 -space by reducing the problem to one concerning convex sets. The third section deals with problems in which convexity appears as a natural restriction on the class of sets involved, such as the measure of closeness of approximation to a convex set by a convex polygon. Finally, special problems about plane convex sets are considered: sets of constant width, and inequalities connecting a plane convex set and its circumscribing triangles.

Dr. Eggleston's exposition is patient and thorough. But the book is for the specialist rather than for the general mathematical reader. Proofs are inevitably long, because of much complicated detail and some tedious but necessary enumeration of cases. Even the author's craftsmanship cannot always cut clearly through the tangle of detail. But there are many unsolved problems in this field, and the young research worker who has a taste for this type of patient analysis will find Dr. Eggleston's books invaluable, both as an account of the present state of knowledge and as a guide to likely lines of advance.
T. A. A. Broadbent 